\title{
Maximum Entropy Method Applied to Time-Series \\ Data in Real-Time Time-Dependent Density Functional Theory ${ }^{\dagger}$
}

\author{
Yasunari Zempo ${ }^{1}$ and Satoru S Kano ${ }^{2}$ \\ 1 Hosei University, Tokyo, Japan \\ 2 Computer and Information Sciences, Hosei University, Tokyo, Japan \\ + Presented at the Entropy 2021: The Scientific Tool of the 21st Century, 5-7 May 2021; Available online: \\ https://sciforum.net/conference/Entropy2021/.
}

Published: 5 May 2021

Density functional theory (TDDFT) in real-time, which is expected to be a key technique to calculate optical spectra. We solve the time-dependent equation, keeping track of the dipole moment as time-series data. In the traditional optical analysis, the oscillator strength distribution is related to the imaginary part of the polarizability, which is usually calculated by the Fourier transform (FT) of the dipole moment. Theoretically, it works only if the time-series data is quite large.

To obtain spectra of fairly high resolution with a relatively small number of time-series data, we have recognized that, from our analysis, even simple MEM provides the oscillator strength distribution at high resolution even with a half of the evolution time of a simple FT. In the practical optical analysis, we are much interested in the lower energy region near the band gap to obtain photo absorption and emission spectra. However, long enough time-evolution is still required in the calculation.

We propose that, as a further improved MEM analysis, we use the concatenated data set made from the several-times repeated raw data. In this process, we also introduce an appropriate phase for the target peak frequency to reduce the side effect of the artificial periodicity. In this procedure, we can effectively take into account the information from large lags of autocorrelation, which represent the interesting signal in the lower energy region.

We have compared the result of our improved MEM and that of FT for the analysis applied to the time-series data in the spectrum analysis of small-to-medium size molecules. As a result, we can observe the clear spectrum of MEM. Our new technique provides higher resolution in fewer time steps, compared to that of FT.

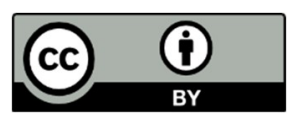

(C) 2021 by the authors. Licensee MDPI, Basel, Switzerland. This article is an open access article distributed under the terms and conditions of the Creative Commons Attribution (CC BY) license (http://creativecommons.org/licenses/by/4.0/). 